Accretion Phenomena and Related Outflows, IAU Colloquium 163

ASP Conference Series, Vol. 121, 1997

D.T. Wickramasinghe, L. Ferrario, and G.V. Bicknell, eds.

\title{
The nature of V Sagittae
}

\author{
Janet H. Wood and J.J. Lockley \\ Department of Physics, Keele University, Keele, ST5 5BG, UK
}

\begin{abstract}
We discuss previous models of V Sge and, in light of our new phase resolved spectroscopy data, suggest a new model: a colliding wind.
\end{abstract}

\section{Introduction}

V Sge is an eclipsing binary with orbital period $12.34 \mathrm{hrs}$. It is currently classified as a novalike variable (e.g. Ritter 1990). The primary eclipse is defined as the deeper eclipse, where the lower-mass, hotter star is eclipsed. In addition to night-to-night variations of up to 0.25 mags, V Sge shows high and low states. High states ( $V=10.0-11.7$ mag outside eclipse) last $\sim 200 \mathrm{~d}$, low states $(\mathrm{V}>$ 12.0) are somewhat shorter (Honneycutt 1996, private communication). The eclipses are shallower in the high state than the low state.

There are dramatic differences in the spectroscopic behaviour during the high and low states (Herbig et al. 1965). In the low state two component $H$, He and sharp OIII fluorescence lines are seen, from which the radial velocities of the two stars can be determined. The secondary star has the stronger HeII emission in the low state. In the high state the OIII lines disappear and the primary dominates the $\mathrm{H}$ and He emission lines, whose widths increase from $400 \mathrm{~km} \mathrm{~s}^{-1}$ to $\sim 1500 \mathrm{~km} \mathrm{~s}^{-1} \mathrm{HWZI}$.

We dearchived HST UV spectroscopy which was taken during a high state. The NV and CIV doublets show P Cygni profiles indicating the presence of a wind. There are narrow absorption components in the NV, CIV and HeII 1640 lines. These were also seen by Herbig et al. (1965) in the optical lines during the low state. They used the radial velocities of these absorption components to map the gas doing the absorbing, deducing that they were caused by material ejected from the primary which continues to rotate with the system.

There have been several models developed for V Sge. Herbig et al. (1965) used their radial velocities together with the depths of the eclipses to constrain the mass ratio and the radii of the stars. They found that the primary star, $\left(0.74 \mathrm{M}_{\odot}\right)$, was close to filling its Roche lobe but the highly evolved, $\left(2.8 \mathrm{M}_{\odot}\right)$, secondary star was well within its Roche lobe.

Williams et al. (1983) obtained spectroscopy through primary eclipse in the high state. They pointed out that the observed changes in the line profiles through the eclipse were similar to the "rotational disturbance" expected from the eclipse of an accretion disc. The duration of the disturbance was unusually long they but explained this as possibly due to the presence of a dense wind around the secondary star. The maximum width of the emission lines indicated that the primary star at the centre of the disc is a white dwarf. Note that in 
this model the secondary star fills its Roche lobe which, given the mass ratio, would lead to unstable mass transfer.

Koch et al. (1986) deduced from IUE data that both components must be contributing to the UV light. They required the helium primary star to fill its Roche lobe. Koch et al. deduced that, if the observed systematic redshift seen in the UV spectroscopy is a gravitational redshift, the secondary component must be a neutron star embedded in a small disc.

Mader (1996) has modelled UBV light curves of V Sge in the high and low states using semi-detached, detached and contact models. They obtained good fits only for a semi-detached configuration with the primary star filling its Roche lobe. The secondary star was very close to filling its Roche lobe. An uneclipsed source of light was required to fit the high state.

Lockley et al. (1996) have fit simultaneous UBV light curves in the low state and found that the primary star is close to or possibly filling its Roche lobe with the secondary star filling $75 \%$ of its Roche lobe (by volume).

Clearly some of these models are mutually incompatible. The models of Herbig et al. (1965), Mader (1996) and Lockley et al. (1996) are similar to each other but not the neutron star model of Koch et al. (1986) or the cataclysmic variable model of Williams et al. (1983). However, Koch's model would require an orbital inclination of only $10^{\circ}$ which is incompatible with the presence of eclipses. The neutron star model for the secondary star cannot therefore be correct. In the next section we present phase resolved spectroscopy around the complete orbit of V Sge. As our new data shows, the variability of the emission lines seen by Williams et al. continues all the way around the orbit and is actually unrelated to the eclipse. It is therefore not a rotational disturbance, and there is no evidence for the presence of a disc or a white dwarf and hence no requirement for the secondary star to fill its Roche lobe.

\section{Phase Resolved Spectroscopic Observations}

We obtained spectroscopy at $1 \AA$ resolution covering HeII 4686 and $\mathrm{H} \beta$ over the complete orbital cycle of V Sge in its high state. Fig. 1(a) shows a trailed spectrum of the HeII line after normalising the continuum and Fig. 1(b) a sequence of spectra around the orbit. The line profiles show highly complex behaviour. The lines have extremely steep sides and several peaks which vary in intensity. Except for the stationary nebular components all the components of the lines have radial velocities phased with the primary star. The nebula line is offset from the centre of the steep sided component. The enhancement of the nebula line during primary eclipse is due to the normalisation. The red peak is maximum and the blue peak minimum at $\phi \sim 0.7$ (where $\phi$ is the orbital phase with $\phi=0.0$ defined by primary eclipse). Each peak is visible for about half the orbit. The middle peak appears at $\phi \sim 0.6$ and vanishes again at $\phi \sim 0.1$. There is also a peak with extremely small radial velocity close to the nebula line. Even this peak is phased with the primary star. There is no obvious eclipse of any individual component of the lines, though there could be an overall decrease in line flux. There is remarkable similarity between the profiles of HeII 4686 and $\mathrm{H} \beta$ around the orbit. Their equivalent widths (EW) behave in exactly the same way through eclipse. On the other hand the behaviour of EW of the CIII/NIII 


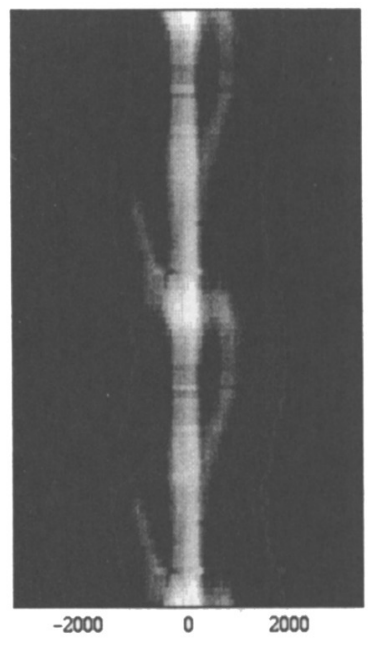

Velocity $(\mathrm{km} / \mathrm{s})$

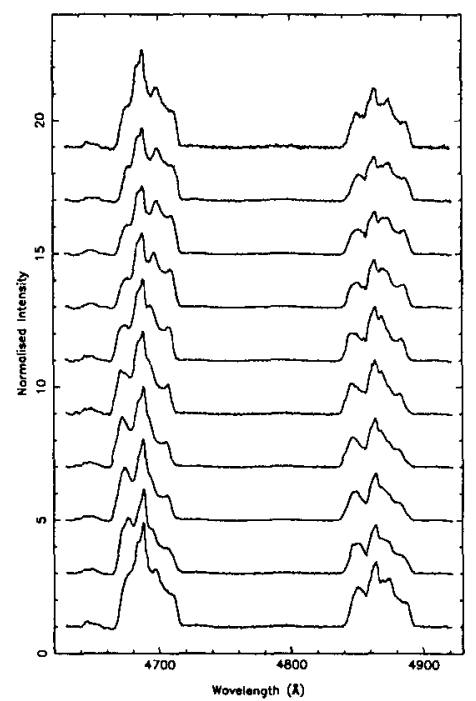

Figure 1. Left: Trailed spectrum of HeII 4686 covering two orbital cycles. $\phi=0.0-2.0$ (upwards). Right: Offset, phase binned spectra through the orbital cycle. Mean $\phi=0.05-0.95$ (upwards).

blend just bluewards of the HeII line shows that it is far more deeply eclipsed at primary minimum than the HeII or $\mathrm{H} \beta$ lines.

We measured the radial velocities of the steep edges of the HeII line using the double Gaussian method, with the separation between Gaussians chosen to match the edges $\left(\sigma=2600 \mathrm{~km} \mathrm{~s}^{-1}\right)$. Fig. 2 (top panel) shows the radial velocities fitted with a model which has $K=225 \mathrm{~km} \mathrm{~s}^{-1}$, much smaller than Herbig's measurement of $K_{1}=320 \mathrm{~km} \mathrm{~s}^{-1}$ based on the OIII line. The maximum occurs at $\phi \sim 0.85$. We also measured the radial velocities of the red and blue peaks (Fig. 2). These are clearly not sinusoidal.

Though the model with the primary close to or filling its Roche lobe and a large secondary star can explain the light curves, it cannot explain the spectroscopic observations in the high state. The very steep sides of the lines would be difficult to achieve even with an accretion disc model. There must be an additional emission line source in the high state to account for the wide, steep-sided lines and the variable peaks. Steep-sided, double-peaked emission lines have been seen in colliding wind systems e.g. V1016 Cyg (Wallerstein et al. 1984). In particular, Lührs (1995) modelled the CIII line in WR 79. WR 79 has the same phasing of the radial velocity and intensity variations of the red and blue peaks as our V Sge data. We therefore suggest that V Sge is actually a colliding wind system with emission from a shock cone, produced by the collision of the winds, producing the lines in the high state. As in Lührs model of WR 79, the cone would be skewed away from the line of centres due to the rotation of the binary.

The line profiles in V Sge are, however, more complex than those in known colliding wind systems. The middle peak and the low velocity peak must be 

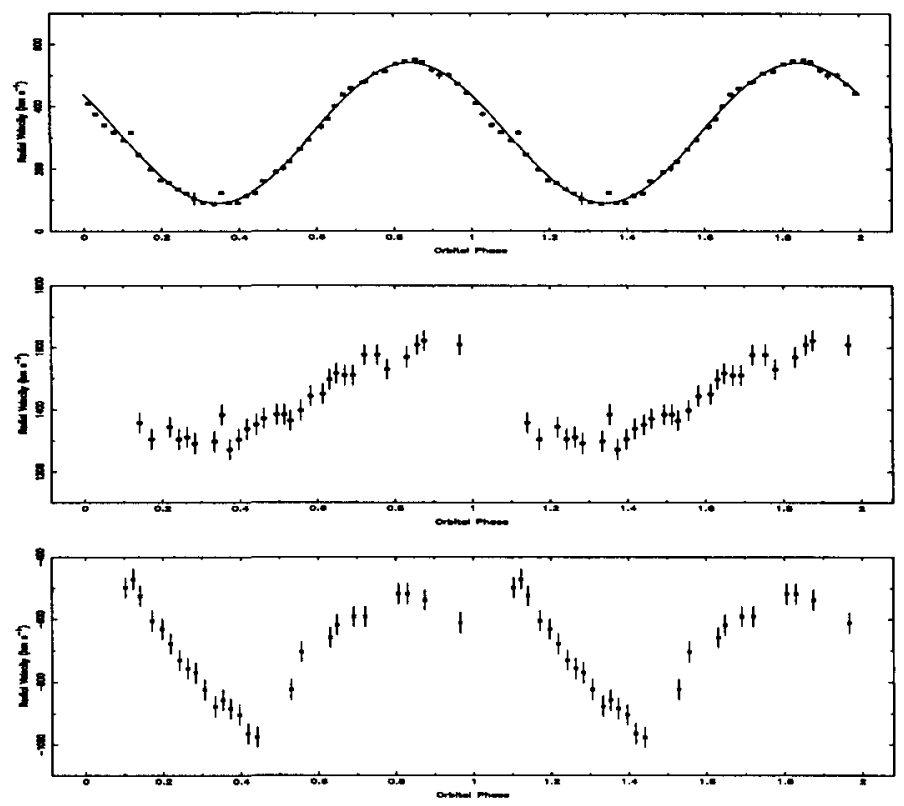

Figure 2. Radial velocities of the HeII 4686 line. Top: the steep edges, Middle: the red peak, Bottom: the blue peak.

produced by another source. We speculate that these peaks are due to irradiation of the primary star (middle peak) and of the secondary star (low velocity peak) by the apex of the shock cone which is offset from the line between the two stars. Since the secondary star is large the centre of mass of the binary lies inside it and hence an area of emission on the secondary star would have the same phasing as the primary star but a low velocity. A full investigation of the colliding wind model in V Sge will be presented in Lockley, Wood \& Eggleton (1996).

Acknowledgments. We thank P. Eggleton for observing assistance.

\section{References}

Herbig, G.H., Preston, G.W., Smak, J., Paczynski, B., 1965, Ap.J., 141, 617

Koch, R.H., Corcoran, M.F., Holenstein, B.D., McClusky, G.E., 1986, Ap.J., 306, 618

Lockley, J.J., Wood, J.H., Eggleton, P.P., 1996, in preparation.

Lockley, J.J., Wood, J.H., Shugarov, S., Naylor, T., Catalan, M.S., 1996, to be submitted to MNRAS.

Lührs, S., 1995, in "Wolf-Rayet Stars, Binaries, Colliding Winds and Evolution", eds. K.A. van der Hucht, P.M. Williams, p416

Mader, J., 1996, MSc thesis, San Diego State University.

Ritter H., 1990, A\&AS, 85, 1179.

Wallerstein, G., Willson, L.A., Salzer, J., Brugel, E., 1984, A\&A, 133, 137

Williams, G., King, A.R., Uomoto, A.K., Hiltner, W.A., 1983, MNRAS, 219, 809 